\title{
INFERRING WINS, DRAWS AND LOSSES FROM POINTS SCORED IN A SPORTS TOURNAMENT
}

\author{
David McSherry
}

\section{Introduction}

International football tournaments like the World Cup often begin with a first round in which teams compete within groups of four for entry to the knockout stage of the tournament. Each team plays each of the other teams in the same group once. A team scores three points for winning a match, one point for a draw, and no points if it loses. In other sporting competitions, the number of teams in a group may differ but is never less than three. The problem of interest in this paper is that of inferring the numbers of matches won, drawn and lost by each team from the points scored by the teams in a group of any size $n \geq 3$.

If $T_{1}, T_{2}, \ldots, T_{n}$ are the teams in a given group $G$, then for $1 \leq i \leq n$, we denote by $p_{i}$ the number of points scored by $T_{i}$ and by $w_{i}, d_{i}$ and $l_{i}$ respectively the numbers of matches won, drawn and lost by $T_{i}$. Conditions or constraints which the inferred values of $w_{i}, d_{i}$ and $l_{i}$ must satisfy include:

$$
\begin{aligned}
& 0 \leq w_{i}, d_{i}, l_{i} \leq n-1 \\
& w_{i}+d_{i}+l_{i}=n-1 \\
& 3 w_{i}+d_{i}=p_{i} \\
& \left(\sum_{i=1}^{n} d_{i}\right) \equiv 0 \bmod 2 \\
& \sum_{i=1}^{n} w_{i}=\sum_{i=1}^{n} l_{i}
\end{aligned}
$$


Constraints (4) and (5) follow from the fact that each match in a given group contributes either two to the total number of draws or one to the total number of wins and one to the total number of losses. Alternatively, constraint (4) can be shown to follow from constraints (2) and (5). The existence of a unique solution to the problem of inferring the wins, draws and losses from the points scored by each team is considered for groups of less than five teams in the following section and for groups of five or more teams in Section 3.

\section{Groups of less than 5 teams}

Where the number of teams in a group is $n=3$, the numbers of wins, draws and losses for a team can always be uniquely determined from the total points $(6,4,3,2,1$, or 0$)$ it has scored in its two matches. For example, a team can score 6 points only if it wins both its matches. In most cases for $n=4$, a team's wins, draws and losses can be uniquely determined without reference to the points scored by other teams in the group. For example, a team can score 7 points only if it wins two and draws one of its matches. Of the total points that a team can score in three matches $(9,7,6,5,4,3,2,1$, or 0$)$, only one can occur in more than one way. A team can score 3 points either by winning one match and losing two or by drawing all three of its matches.

Provided there is only one team with 3 points, a simple rule based on constraint (4), according to which the total number of draws must be even, can be used to determine whether a team with 3 points has three draws or a win and two losses. For example, if the points scored are 5, 5, 3 and 2, then the team with 3 points cannot have three draws, since the total number of draws for the other three teams is $2+2+2=6$. Unfortunately this rule does not provide a straightforward solution if more than one team has three points. However, as the following theorem shows, the possible ways of scoring 3 points in a group of 4 teams can always be distinguished regardless of the number of teams with 3 points. For example, if the points scored are 5, 3, 3 and 2, then according to the theorem, each of the teams with 3 points has three draws. 
Theorem 2.1 Let $M$ be the total of the points scored by all the teams in the group. If any team $T$ in a group of four teams has scored three points, then the wins, draws and losses for $T$ can be determined as follows:

1. if $M>15$, then $T$ has one win and two losses;

2. if $M<15$, then $T$ has three draws;

3. if $M=15$, then $T$ has three draws if and only if one of the other teams has scored 4 points.

Proof: It can be seen that $M=3+M_{1}+M_{2}$, where $M_{1}$ is the total of the points scored by the other three teams from their matches with $T$, and $M_{2}$ is the total of the points scored by the other three teams from the matches in which $T$ is not involved. It can also be seen that $6 \leq M-2 \leq 9$, as each of the three matches in which $T$ is not involved contributes 2 or 3 points to $M_{2}$.

If $M>15$, then $M_{1}+M_{2}>12$ and so $M_{1}>12-M_{2} \geq 3$.

So $T$ cannot have three draws and therefore has a win and two losses. If $M<15$, then $M_{1}+M_{2}<12$ and so $M_{1}<12-M_{2} \leq 6$. So $T$ cannot have lost two matches, and therefore has three draws. If $M=15$ and $T$ has three draws, then $M_{1}=3$ and so $M_{2}=9$. The points scored by the other teams in the matches not involving $T$ must therefore be 6,3 and 0 or 3,3 and 3 . At least one of the other teams must therefore have a total of 4 points.

On the other hand, if $M=15$ and $T$ does not have three draws, then $T$ must have lost 2 matches and so $M_{1}=6$. Thus $M_{2}=6$ and so the three matches in which $T$ was not involved were draws. Each of the other teams must therefore have a total of 2 or 5 points.

An immediate corollary of Theorem 2.1 is that it is not possible for two teams in a group of four teams to score 3 points in different ways.

\section{Groups of 5 or more teams}

It is shown in this section that for a group $G$ of any size $n \geq 5$, there is at least one possible set of points totals for which the numbers of wins, draws and losses for each team in $G$ can be uniquely determined. On the other hand, there is also a possible 
set of points totals for which the wins, draws and losses cannot be uniquely determined for every team.

Proposition 3.1 For any group $G=\left\{T_{1}, T_{2}, \ldots, T_{n}\right\}$, the following are equivalent:

(a) for $1 \leq i \leq n, p_{i}=3(n-i)$;

(b) for $1 \leq i \leq n, T_{i}$ beat all its successors in $G$, if any, and was beaten by all its predecessors, if any, in $G$;

(c) for $1 \leq i \leq n, w_{i}=n-i, d_{i}=0$ and $l_{i}=i-1$.

Proof: It is easily seen that (b) implies (c) implies (a). Given that (a) is true, $T_{1}$ has $3(n-1)$ points and must therefore have beaten all its successors in $G$. As $T_{1}$ has no predecessors in $G$, the proposition stated in (b) to be true for $1 \leq i \leq n$ is therefore true for $i=1$. Now assume the proposition true for $1 \leq i \leq k$, where $k \leq n-1$, and consider the case of $T_{k+1}$. By assumption, $T_{k+1}$ was beaten by $T_{i}$ for $1 \leq i \leq k$ and yet by (a), $T_{k+1}$ has scored $3(n-k-1)$ points. Thus $T_{k+1}$ must have beaten all its successors, if any, in $G$. It follows by induction that the proposition stated in (b) is true for $1 \leq i \leq n$, so (a) implies (b).

Proposition 3.2 For any group $G=\left\{T_{1}, T_{2}, \ldots, T_{n}\right\}$, where $n \geq$ 5 , there is a possible set of points totals for which the wins, draws and losses cannot be uniquely determined for every team in $G$.

Proof: Suppose that the points scored by $T_{1}, T_{2}, T_{3}, T_{4}$ and $T_{5}$ are $10,4,4,4$ and 4, respectively, and that for $6 \leq i \leq n$, if any, $T_{i}$ has scored $3(i-1)$ points. By an argument similar to that used in the proof of Proposition 3.1, it can be inferred that for $6 \leq i \leq n$, if any, $T_{i}$ must have beaten all its predecessors in $G$ and been beaten by all its successors, if any, in $G$. Each of $T_{1}, T_{2}, T_{3}, T_{4}$ and $T_{5}$ therefore has at least $n-5$ losses.

To have scored 10 points in spite of its $n-5$ losses, $T_{1}$ must have won three and drawn one of its matches with $T_{2}, T_{3}, T_{4}$ and $T_{5}$. If, for example, $T_{1}$ drew with $T_{2}$, then the points scored by $T_{2}$, $T_{3}, T_{4}$ and $T_{5}$ in the matches involving only these four teams must be 3, 4, 4 and 4 respectively. By Theorem 2.1, $T_{2}$ must therefore have drawn with $T_{3}, T_{4}$ and $T_{5}$ as well as with $T_{1}$. To have scored 4 points, each of $T_{3}, T_{4}$ and $T_{5}$ must have one win in addition to 
its draw with $T_{2}$. One possible scenario is that $T_{3}$ beat $T_{4}, T_{4}$ beat $T_{5}$, and $T_{5}$ beat $T_{3}$. Outcomes for all the matches in $G$ have thus been identified which are consistent with the points scored by each team and according to which $T_{2}$ has four draws and each of $T_{3}, T_{4}$ and $T_{5}$ has one draw. Since $T_{1}$ may have drawn instead with $T_{3}, T_{4}$ or $T_{5}$, four possible sets of match outcomes, in each of which a different team has four draws, can be seen to exist. So the wins, draws and losses for $T_{2}, T_{3}, T_{4}$ or $T_{5}$ cannot be uniquely determined in this case.

\section{Conclusions}

The wins, draws and losses for each team in a group of three or four teams can always be uniquely determined from the points scored by each team in the group. For such a group, much of the information typically provided in the final group table (wins, draws and losses, goals for and against, and points scored by each team) is therefore redundant. A significant reduction in the space required for storage of the group results in a database is therefore possible without loss of information. For groups of more than four teams, however, the wins, draws and losses for each team cannot in general be discarded as there is always a possible set of points totals for which they cannot be uniquely determined.

A problem related to that of inferring the wins, draws and losses for each team in a given group is that of inferring the score in each match form the points scored and total numbers of goals for and against each team. Although a unique solution to the latter problem is not always possible even for groups of three or four teams, interesting results have been obtained by an approach based on constraint satisfaction techniques, [1, 2], and will be reported in detail elsewhere.

\section{References}

[1] S. J. Russell and P. Norvig, Artificial Intelligence: A Modern Approach. Prentice-Hall: New Jersey, 1995. 
[2] E. C. Freuder and R. J. Wallace, Partial constraint satisfaction, Artificial Intelligence 58 (1992), 21-70.

David McSherry

School of Information and Software Engineering

University Of Ulster

Newtownabbey

County Antrim BT37 0QB

Northern Ireland 